\title{
Intervenção comportamental para treinadores no uso do estabalecimento de metas no tênis ${ }^{1}$
}

\author{
Behavioral intervention for coach in the \\ use of goal setting in tennis
}

\author{
Camila Harumi SUDO² \\ Silvia Regina de SOUZA ${ }^{2}$
}

\begin{abstract}
Resumo
O trabalho avaliou os efeitos da capacitação de um treinador de tênis para o uso de estabelecimento de metas. A pesquisa consistiu em três fases. Na linha de base (Fase 1) filmaram-se 11 sessões de treino, sem interferência da pesquisadora. A intervenção (Fase 2) consistiu em 10 sessões nas quais se ensinou ao treinador como observar e registrar comportamentos-alvo, planejar critérios de proficiência para suas ocorrências e fornecer feedback propondo mudanças quando necessárias. A pós-intervenção (Fase 3) foi semelhante, em estrutura, à linha de base. Ao longo das fases, verbalizações e outros comportamentos do treinador (registrar, participar e variar exercícios) foram registrados e categorizados. Verificou-se um aumento na porcentagem de instruções, correções, elogios descritivos, registros dos desempenhos dos atletas e participação do treinador nos exercícios, bem como redução na porcentagem de críticas/sarcasmos, durante e após a intervenção. Os comportamentos do treinador generalizaram-se para outros contextos, e os atletas alcançaram suas metas. Os resultados instigam novas discussões.
\end{abstract}

Unitermos: Análise do comportamento. Capacitação de treinadores. Estabelecimento de metas. Psicologia do esporte.

\begin{abstract}
The study evaluated the effects of training a tennis coach in the use of goal setting and consisted of three phases. At baseline (Phase 1), 11 training sessions without interference from the researcher were recorded. The intervention (Phase 2) consisted of 10 sessions in which the coach was taught how to observe and record the target behaviors, plan criteria for proficiency of the occurrences and provide feedback by proposing changes when necessary. The post-intervention (Phase 3) was similar in structure to the pretest. Throughout the phases, verbalizations and other behaviors of the coach (recording, participating and varying exercises) were recorded and categorized. An increase was noted in the percentage of instructions, corrections, descriptive compliments, records of athletes' performance and the coach's participation in the exercises, and a reduction in the percentage of critical/sarcastic attitudes throughout and after the intervention. The coach's behavior became generalized into other contexts and athletes reached their goals. Our results call for further discussion.
\end{abstract}

Uniterms: Behavior analysis. Coach training. Goal setting. Sport psychology.

$\boldsymbol{\nabla \nabla} \boldsymbol{\nabla} \boldsymbol{\nabla}$

1 Artigo elaborado a partir da tese de C.H. SUDO, intitulada "Avaliação de um programa de capacitação de treinadores para a utilização de estabelecimento de metas no esporte". Universidade Estadual de Londrina, 2008.

2 Universidade Estadual de Londrina, Centro de Ciências Biológicas, Departamento de Psicologia Geral e Análise do Comportamento. Rod. Celso Garcia Cid, PR 445, Km 380, Caixa Postal 6001, 86051-990, Londrina, PR, Brasil. Correspondência para/Correspondence to: S.R. SOUZA. E-mails: <ssouza@uel.br>; <ssouza_ssouza@hotmail.com>.

Agradecimento: À Fundação Araucária. 
Estudos têm investigado a influência de comportamentos de treinadores sobre seus atletas (Brandão \& Carchan, 2010; Mallett \& Côté, 2006; Martens, 1990) e destacado a importância da formação e capacitação desses profissionais (Coatsworth \& Conroy, 2006; Cruz, Torregrosa, Sousa, Mora \& Viladrich, 2011; Cunha, Mesquita, Rosado, Sousa \& Pereira, 2010; Mora, Cruz \& Torregrosa, 2009; Ramos, Graça, Nascimento \& Silva, 2011; Smith \& Smoll, 2011; Smoll \& Cumming, 2007).

Cruz et al. (2011) afirmam que há muitos programas de capacitação de treinadores sendo desenvolvidos, a maioria dos quais oferece poucas oportunidades para eles aplicarem o conteúdo aprendido e receberem feedback de seu desempenho. Os programas são, em geral, meramente informativos, inflexíveis às necessidades reais dos treinadores, além de ser difícil identificar medidas comportamentais específicas para a avaliação de seus resultados (Cruz et al., 2011; Gilbert \& Trudel, 1999; Smith \& Smoll, 2011). Além disso, ao avaliar o impacto que tais intervenções produzem nos atletas, os estudos usam medidas indiretas, como autoestima (Coatsworth \& Conroy, 2006) e ansiedade (Smith et al., 2007) etc., obtidas a partir do autorrelato dos atletas, e não do registro sistemático de seus comportamentos.

Dos programas de capacitação de treinadores citados na literatura, destacam-se, por sua relevância na área, os de Smoll, Smith e Curtis (1983), Smith, Smoll e Hunt (1977) e Cruz et al. (2011). Smoll et al. (1983) e Smith et al. (1977) desenvolveram o Programa de Formação para a Eficácia dos Treinadores (PFET), constituído por um workshop de 3 horas no qual treinadores são instruídos sobre a importância de recompensar tanto esforços quanto resultados, de corrigir desempenhos sem o uso de punições, de dar modelos de comportamentos desejados, e de criar condições para a aprendizagem de novas habilidades. Ressalta-se o uso de procedimentos como role-playing e modelação, e o encorajamento dos treinadores a monitorarem seus comportamentos, recebendo feedback desses registros. Esses procedimentos pareciam facilitar que o aprendizado durante o workshop se generalizasse para contextos reais de treino e competição. Também os comportamentos de treinadores submetidos a esse tipo de intervenção eram, frequentemente, mais bem avaliados 564 pelos atletas.
Em outro estudo, Cruz et al. (2011) investigaram a efetividade do Programa de Asesoramiento Personalizado a Entrenadores (PAPE). Neste, os próprios participantes são encorajados a escolher os comportamentos-alvo que desejam mudar, a partir da análise de categorias comportamentais registradas com o auxílio do Coaching Behavior Assessment System (CBAS), um instrumento de observação sistemática elaborado por Smith et al. (1977) e empregado em muitos estudos da área (Conde, Almagro, Saenz-Lopez\& Castilho, 2009; Cruz et al., 2001; Mora et al., 2009; Sousa, Cruz, Torregrosa, Vilches \& Viladrich, 2006). Como resultado, tais estudos têm mostrado mudanças significativas nos comportamentos-alvo escolhidos pelos treinadores, quando comparados com os de participantes que não escolheram suas metas.

Em outro exemplo, Demmers, Woodburn e Savard (2006) utilizaram um modelo de formação de treinadores em ambiente simulado de ensino. Os aprendizes eram encorajados a planejar as aulas juntos, a ministrarem-nas a seus atletas, a se autoavaliarem e a receberem feedback de seus pares, após as aulas. Nas aulas seguintes, eles recebiam feedback imediato de um profissional formado, mediante ponto auditivo. Depois, trabalhavam em pares e, enquanto um ensinava, o outro providenciava feedback, por meio de ponto auditivo, para o colega, com a supervisão de um professor. Por fim, os aprendizes eram encorajados a realizar um estágio sob a supervisão de um professor. Porém, Demmers et al. (2006) sugeriram que, ao final do programa, fossem avaliados os comportamentos dos futuros treinadores.

Apesar de os estudos citados acima usarem procedimentos (autorregistro, feedback, modelação etc.) que podem facilitar a generalização dos comportamentos do treinador para outros contextos, analisar medidas observáveis de comportamento e oferecer atendimento mais personalizado, eles ainda apresentam fraquezas. Por exemplo, alguns preveem apenas duas sessões de linha de base (Mora et al., 2009; Sousa et al., 2006), o que, segundo Kazdin (1982), não permite ao pesquisador avaliar a estabilidade do comportamento ao longo do tempo nem descartar tendências, na linha de base, que sugiram a interferência de outras variáveis além da intervenção. Verifica-se que se, por um lado, há estudos interessados no aperfeiçoamento de metodologias e análises, por outro, mais investigações ainda são necessárias. 
Considerando-se a possibilidade de capacitar treinadores em habilidades específicas, eles poderiam ser ensinados a usar a estratégia de estabelecimento de metas. Essa tem sido apontada como uma importante estratégia para a aprendizagem de novas habilidades (Corrêa \& Souza Júnior, 2009; Marinho, 2009; Neiva, 2010) e melhora de desempenhos atléticos já estabelecidos (Ward, 2011; Weinberg \& Gould, 2001), mas os treinadores demonstram não saber como usá-la (Burton, Weinberg, Yukelson \&Weingand, 1998).

Uma meta define-se como"... um nível de desempenho em direção ao qual um indivíduo ou grupo deve trabalhar" (Martin, 2001, p.44); ou "... um nível específico de domínio de uma tarefa, dentro de um intervalo de tempo estabelecido antecipadamente" (Gonzáles, 2001, p.98). Conforme referencial teórico da análise do comportamento, estabelecer metas envolve descrever verbalmente um comportamento, as condições nas quais ele deve ocorrer, bem como suas possíveis consequências. Isso é especialmente útil ao alterar as chances de um comportamento ocorrer, independentemente de tentativas e erros (Martin, 2001; Ward, 2011).

Para aumentar a efetividade das metas, estudos sugerem que: a) metas de resultado como "vencer um jogo" são úteis apenas se envolverem metas de desempenho, como "aumentar em 50\% a frequência de acertos" (Mellalieu, Hanton \& O'Brien, 2006; Weinberg \& Gould, 2001); b) metas devem ser difíceis, porém realistas, conforme o nível de desempenho inicial do atleta (Corrêa \& Souza Júnior, 2009; Swain \& Jones, 1995; Ugrinowitsh \& Dantas, 2002); c) objetivos muito distantes devem ser subdivididos em prazos menores (Gonzáles, 2001); d) metas difíceis devem ser divididas em metas simples, principalmente no aprendizado de comportamentos complexos (Getz\& Rainey, 2001); e) é útil fornecer consequências para o cumprimento das metas, mesmo que de forma arbitrária: elogios, prêmios (Brobst \& Ward, 2002; Gamba, 2007; Souza, Sudo, Gamba, Oliveira \& Teixeira, 2009; Sudo \& Souza, 2009), assim como é importante descrever as consequências do não cumprimento das metas (Ward, 2011) ef) envolver os atletas no estabelecimento de suas metas pode aumentar seu comprometimento com o programa (Mellalieu et al., 2006; Ward \& Carnes, 2002).

Enfatiza-se que o uso de metas mais simples, de prazos menores, e o fornecimento de consequências facilitam o aprendizado (Sudo \& Souza, 2009). Para a análise do comportamento, o conceito de modelagem representa adequadamente esse processo pelo qual habilidades mais complexas podem ser ensinadas, partindo-se do reforço da execução de seus componentes mais simples (Skinner, 1953). Pesquisas têm demonstrado a efetividade da modelagem no estabelecimento de metas para atletas (Brobst \& Ward, 2002; Cedra \& Sério, 2008; Mellalieu et al., 2006).

Dentre os estudos sobre essa questão, destaca-se o de Gamba (2007), que investigou os efeitos do estabelecimento de metas e feedback na execução do salto Tsukahara realizado por cinco meninos praticantes de ginástica artística, com idade entre 8 e 10 anos. Inicialmente, o treinador foi auxiliado a escolher um comportamento-alvo (salto), dividi-lo em componentes (entrada de lado, repulsão, cair fechando e rolar e mortal) e criar sete submetas, conforme desempenho inicial de cada atleta. Na intervenção, o pesquisador mostrou aos atletas as submetas estabelecidas e explicitou que placas de cortiça seriam usadas como feedback de seu desempenho. Cumpridas as submetas, os atletas poderiam escolher uma recompensa (cinema, carrinhos etc.) oferecida pelo pesquisador. Cada vez que o atleta avançasse uma submeta, o pesquisador fornecia novas instruções. Os resultados mostraram que três participantes conseguiram alcançar as submetas propostas e que houve aumento não só do número de componentes realizados corretamente, mas também da porcentagem de execuções corretas consecutivas.

Não obstante os resultados obtidos por Gamba (2007), alguns aspectos de seu estudo precisam ser considerados, como o fato de que foram necessárias várias sessões de intervenção, aumentando a interferência da prática e do tempo, e não foi possível alcançar estabilidade da linha de base com todos os participantes, impossibilitando afirmativas quanto à efetividade do programa. A despeito disso, o procedimento criou condições para que consequências que seriam possíveis somente em longo prazo estivessem presentes em curto prazo (Getz \& Rainey, 2001; Gonzáles, 2001).

Outros estudos avaliaram os efeitos do estabelecimento de metas sobre repertórios atléticos já estabelecidos (Mellallieu et al., 2006; Swain \& Jones, 1995) e revelaram que tão importantes quanto a descrição da 
meta são as consequências do seu cumprimento. Quanto mais reforçadores naturais estiverem presentes, maior a chance de manutenção do aprendizado (Martin, 2001), assim como com o uso de reforçadores arbitrários (Brobst \& Ward, 2002; Hume \& Crossman, 1992).

Constata-se que o estabelecimento de metas envolve uma série de passos, embora muitas pesquisas não os detalhem. Ele implica realizar observações e registros sistemáticos, coletar dados de linha de base de comportamentos-alvo, programar etapas de evolução do desempenho desses, consequenciar progressos e fornecer feedbacks precisos e contingentes a eles. Ou seja, faz-se necessário conhecer as contingências presentes na determinação desses comportamentos-alvo, condição cuja falta tem sido apontada como uma das principais falhas nos estudos sobre o estabelecimento de metas no esporte (Ward, 2011).

Assim, a efetividade do estabelecimento de metas pode estar relacionada, também, à forma como os aplicadores têm conduzido a sua utilização. Nessa linha, o presente estudo avaliou os efeitos da capacitação de um treinador no uso do estabelecimento de metas nos treinos com seus atletas.

\section{Método}

\section{Participantes e Local}

Participou um treinador de tênis de 21 anos de idade e três anos de experiência. O estudo foi conduzido em um clube, durante o treino de dois atletas de 15 e 16 anos, ambos com três anos de treino Trabalho aprovado pelo Comitê de Ética da Universidade Estadual de Londrina, Parecer 304/06, CAAE no 0261.0.268.000-06, emitido em 30 de novembro de 2006. Cada treino durava 1 hora.

\section{Equipamentos e materiais}

Usou-se uma filmadora e um gravador digital para o registro das verbalizações do treinador, materiais impressos para as orientações do mesmo e folhas de papel para registro das ocorrências de comportamentos não verbais do participante.

\section{Classificação dos comportamentos do treinador}

Os comportamentos do treinador, durante os treinos, foram analisados conforme oito categorias comportamentais verbais, elaboradas com base em estudos sobre capacitação de treinadores e sobre estabelecimento de metas (Lima \& Souza, 2009; Rushall \& Smith, 1983; Smith et al., 1977), e quatro categorias comportamentais não verbais, conforme mostra o Quadro 1.

\section{Procedimentos}

Após assinatura do Termo de Consentimento Livre e Esclarecido pelo treinador e seus atletas, deu-se início à pesquisa, que se caracteriza como um estudo de caso. Esta foi conduzida em três fases: linha de base, intervenção e pós-intervenção ${ }^{3}$. Durante todas as fases, as verbalizações do treinador foram gravadas, transcritas e categorizadas, e a porcentagem de ocorrência de cada categoria foi calculada, bem como a quantidade de exercícios aplicados pelo treinador, a porcentagem de exercícios relacionados ao treino de um comportamento-alvo (meta) e a quantidade de treinos nos quais ocorreram comportamentos de registrar e participar do exercício.

Na linha de base foram filmadas 11 sessões de treino, sem nenhuma interferência da pesquisadora, e os resultados expostos em gráficos.

Na intervenção aplicou-se o Programa Comportamental de Capacitação de Treinadores (PCCT) para o uso do estabelecimento de metas, o qual foi dividido em quatro passos (Quadro 2). Para a aplicação do PCCT, o treinador foi orientado a escolher apenas um atleta (A1), a quem os passos deveriam ser aplicados com auxílio da experimentadora. Posterior-mente, ele deveria aplicar os mesmos passos com o outro atleta (A2), sem auxílio. Cada sessão de intervenção durou de 30 a 45 minutos, sendo realizada antes dos treinos. A sessão

$\boldsymbol{\nabla} \boldsymbol{\nabla} \boldsymbol{\nabla}$

3 Dois auxiliares de pesquisa receberam treinamento para que pudessem auxiliar no registro dos dados. Eles foram considerados aptos para a realização 
requeria do treinador que ele aplicasse o que havia aprendido com o atleta A1. O feedback dos comportamentos do treinador - conforme o que fora trabalhado na sessão de intervenção - era fornecido pela pesquisadora, durante e após cada treino.
No primeiro passo, o treinador foi ensinado a fazer registros de topografia, latência e frequência de comportamentos, a partir de recortes de vídeos de diferentes esportes. Em seguida solicitou-se que ele escolhesse e registrasse a ocorrência de um comporta-

Quadro 1. Categorias comportamentais verbais de treinadores e categorias de análise referentes aos tipos de exercícios, aos exercícios relacionados ao treino do comportamento-alvo, aos comportamentos do treinador de registrar e participar dos exercícios.

\begin{tabular}{|c|c|}
\hline & Categorias Comportamentais Verbais \\
\hline Categorias & Descrições \\
\hline $\begin{array}{l}\text { Instruções Técnicas } \\
\text { Parciais (ITP) }\end{array}$ & $\begin{array}{l}\text { O treinador verbaliza o que o atleta deverá fazer durante um } \\
\text { exercício, sem descrever as consequências do comportamento } \\
\text { instruído }\end{array}$ \\
\hline $\begin{array}{l}\text { Instruções com } \\
\text { Descrição de } \\
\text { Consequências (IDC) }\end{array}$ & $\begin{array}{l}\text { O treinador verbaliza o que o atleta deverá fazer durante um } \\
\text { exercício e especifica as consequências naturais ou arbitrárias do } \\
\text { seguimento da instrução fornecida }\end{array}$ \\
\hline Encorajamento (ENC) & $\begin{array}{l}\text { O treinador encoraja os atletas a terminar um exercício e a executá- } \\
\text {-lo de acordo com os critérios ou prazos }\end{array}$ \\
\hline $\begin{array}{l}\text { Críticas ou Sarcasmos } \\
\text { (CS) }\end{array}$ & $\begin{array}{l}\text { O treinador faz críticas aos atletas, responde de modo ríspido a } \\
\text { suas perguntas e/ou utiliza expressões sarcásticas com o atleta }\end{array}$ \\
\hline Correções Parciais (CO) & $\begin{array}{l}\text { O treinador diz ao atleta que existe uma habilidade executada } \\
\text { incorretamente e enfatiza apenas o que o atleta não deve fazer }\end{array}$ \\
\hline $\begin{array}{l}\text { Correções com } \\
\text { Descrição de } \\
\text { Consequências (CODC) }\end{array}$ & $\begin{array}{l}\text { O treinador sinaliza a habilidade executada incorretamente, } \\
\text { descreve o que o atleta não deverá fazer e as consequências } \\
\text { naturais ou arbitrárias desse comportamento }\end{array}$ \\
\hline
\end{tabular}

Elogio Parcial (EP)

Elogio Descritivo (ED)
O treinador verbaliza elogios sem descrever o que o atleta acertou.

O treinador elogia e verbaliza o que o atleta fez corretamente, descrevendo as consequências que ocorreram após o comportamento adequado
"Faz a bola passar a 1 metro e meio da rede ai". "Joga a bola mais no fundo"

"Faz a bola passar a 1 metro e meio da rede aí que ela vem pro fundo". "Procura afastar pra você ganhar o espaço e poder continuar jogando o peso do corpo pra cima"

"Vamos, vamos, vamos". "Só mais um minutinho, vamos lá". "Vai que você consegue"

"Beleza, pode deixar dar mais um pingo mesmo. No jogo também". "Ah, com certeza melhorou. Melhorou muito mesmo"

"Voleio de direita tá muito aqui ó". "Não leva o tronco não". "Para de flexionar o tronco na hora que você for bater de esquerda, volear" "Você está subindo demais. A bola tá passando e você tá pegando ela aqui ó". "Quanto mais você bater só de direita no treino, mais você vai errar a esquerda no jogo"

"Isso. Joia!". "Boa bola!"

"Jogou o peso do corpo legal de novo". "Você viu como essa você pegou bem lá na

frente?"

Treinador fica de um lado da quadra e os atletas do outro, recebendo bolas jogadas pelo treinador 
Quadro 2. Descrição dos objetivos e procedimentos adotados na aplicação do primeiro e segundo passo do programa comportamental de capacitação de treinadores. Londrina (PR), 2006-2007.

\begin{tabular}{lll}
\hline Passos e Objetivos & \multicolumn{1}{c}{ Procedimentos antes do treino } & Procedimentos durante e após o treino \\
\hline Passo 1: Descrição do & Sessão 1 (120 treino) & Após o treino: \\
Comportamento-alvo. & - ensino de registros de topografia, duração, latência e frequência & feedback para o treinador dos seus registros \\
Objetivos: Capacitar o & de comportamentos & do desempenho do atleta A1
\end{tabular}

treinador a: fazer

observação sistemática e

registro do

- orientação para a operacionalização do comportamento-alvo e

registros desse, durante o treino subsequente

comportamento-alvo

Sessão 2 (130 treino)

- feedback da operacionalização e registro do comportamento-alvo de $\mathrm{A} 1$, ocorridos no treino anterior.

Sessão 3 (140 treino)

- feedback do exercício de operacionalizar outros comportamentos

- orientação para o uso de linha de base múltipla entre comportamentos-alvo

Passo 2: Programação

de etapas e critérios de evolução

Objetivos: Capacitar o treinador a: estabelecer passos com critérios e prazos para o treino sistemático do comportamento-alvo

Sessão 4 (150 treino)

- Revisão do passo 1 por meio de leitura de material impresso - orientação para a análise, em gráfico, da linha de base de A1

- orientação para a elaboração das metas

-orientação para treinador dizer ao atleta qual o comportamento -alvo que seria trabalhado

Sessão 5 (16º treino)

- feedback do programa de metas feito pelo treinador

-orientação e modelo de como o treinador poderia apresentar o programa de metas para o atleta $\mathrm{A} 1$

Sessão 6 (170 treino)

- orientação para identificação de antecedentes e consequentes

dos desempenhos desejados de atletas

- orientação sobre a importância de priorizar instruções mais do que correções, dar modelos e de fazer elogios descritivos

- orientação para o uso das filmagens para dar feedback para A1

- orientação para o uso de modelos para que a A1 imitasse

Passo 3: Reforçamento

Positivo.

Sessão 7 (18० treino)

- orientações sobre reforçadores naturais e arbitrários

- feedback das consequências verbais oferecidas pelo treinador

- ensino do uso de feedback visual com os atletas

treinador a identificar

possíveis reforçadores

Sessão 8 (190 treino)

dos comportamentos de

seus atletas e a utilizá-los

em treino

- verificação da lista de recompensas dos atletas e incentivo do treinador para utilizar algumas dessas recompensas

- feedback de algumas recompensas já utilizadas pelo treinador

Sessão 9 (20 treino)

- orientação para a utilização do reforçamento arbitrário, por meio de prêmios oferecidos ao atleta $A 1$, dado o cumprimento da meta

Passo 4: Manutenção. Sessão 10 (210 treino)

Objetivos: Capacitar o - Elogio do comportamento do treinador de trazer, espontatreinador a analisar os

registros do

desempenho do atleta e

a reavaliar metas

neamente, os gráficos do A1 atualizados e três sessões de linha de base do comportamento-alvo de A2

- orientação e modelo quanto à importância de acompanhar o progresso ao longo das metas e de reavaliá-las, se necessários

- orientação do treinador para a aplicação do programa com o outro atleta
Após o treino: feedback dos registros

Após o treino: feedback dos registros; Elogios pelos registros de outros comportamentos de A1

Após o treino: feedback para o treinador sobre seu desempenho de verbalizar para o atleta qual seria o comportamento-alvo trabalhado

Durante o treino: feedback da apresentação das metas para A1. Após o treino: feedback dos registros

Durante o treino: feedback dos elogios descritivos, modelos combinados com instruções, e imitação do modelo fornecido pelo treinador

Após o treino: feedback para o treinador sobre seu desempenho de elogiar acertos e corrigir erros, ao invés de criticar

Após o treino: feedback dos elogios, instruções específicas e descrições de consequências de acertos e erros dos atletas

Nenhum feedback foi dado

Após o treino: feedback para o treinador sobre seu desempenho em verbalizar novos critérios de desempenho para A1 (treinador diminuiu o grau de dificuldade do programa de metas de A1) 
mento (comportamento-alvo) do atleta A1, que precisasse ser melhorado. Durante três sessões, ele fez o registro do comportamento-alvo e estabeleceu, com o auxílio da pesquisadora, a linha de base desse comportamento.

O Passo 2 iniciou-se com uma breve revisão do Passo 1. Depois, ensinou-se o treinador a avaliar a linha de base do comportamento-alvo de A1 e a criar etapas e critérios de evolução desse comportamento; discutiram-se com o treinador condições que poderiam tornar uma meta mais efetiva e exemplos de comportamentos que poderiam ser apresentados por ele (dar modelos, dar instruções e elogios descritivos, mostrar filmagens do atleta etc.), com vistas a auxiliar o atleta na execução do comportamento-alvo. Mostraram-se também ao treinador exemplos de situações antecedentes e consequentes ao comportamento-alvo do atleta que poderiam interferir em seu desempenho, por meio de trechos dos treinos filmados. Por fim, o treinador foi encorajado a expor o programa de metas elaborado por ele, para o atleta.

No terceiro passo, abordou-se a importância de consequenciar as mudanças de um comportamentoalvo. Para isso, usaram-se trechos das gravações das verbalizações do treinador, nos quais ele elogiava, incentivava, punia ou corrigia o comportamento do atleta. Da mesma forma, orientou-se o treinador a fazer o levantamento de possíveis fontes de reforçadores arbitrários, junto ao atleta escolhido, que pudessem servir como premiação ao final do programa.

No último passo, a pesquisadora e o treinador fizeram a revisão do programa de metas, analisando-se o registro do comportamento-alvo, ao longo dos treinos. Em seguida, pediu-se ao treinador que repetisse todos os procedimentos aprendidos durante a intervenção, com o outro atleta (A2), porém sem auxílio da pesquisadora.

Na pós-intervenção, filmaram-se oito sessões de treino, sendo que nenhum feedback foi dado nesse período. Na última sessão, ambos os atletas já haviam alcançado suas metas e, assim, agendou-se uma reunião de entrega dos prêmios e de avaliação do trabalho pelo treinador e atleta. Para a avaliação, utilizou-se um roteiro de entrevista com questões relativas à satisfação dos atletas e do treinador com o programa de metas. As entrevistas foram gravadas e transcritas.

\section{Resultados}

A Figura 1 mostra a porcentagem de cada categoria verbal analisada na interação do treinador com os atletas, ao longo do estudo. A porcentagem de ocorrência de cada categoria foi calculada com base na frequência total de ocorrências de todas as categorias analisadas.

De acordo com a Figura 1, 36,00\% dos comentários feitos pelo treinador, durante a linha de base, referiram-se às instruções fornecidas aos atletas, principalmente as Instruções Parciais (ITP). Com a introdução da intervenção, a porcentagem de Instruções com Descrição de Consequências (IDC) aumentou e a de ITP diminuiu, principalmente a partir das sessões 15 e 16. A porcentagem média de comentários de Encorajamento (ENC) também foi alta $(27,95 \%)$ e, após a intervenção, aumentou ainda mais (31,41\%). Ao contrário, a porcentagem de ocorrência de Críticas/Sarcasmos (CS), durante a linha de base, foi baixa (média de 5,00\%), mas observouse variabilidade desses dados, sendo as ocorrências de CS mais frequentes em algumas sessões (por exemplo, nas sessões 6, 7, 8 e 11). Apesar da instabilidade dos dados não permitir afirmações quanto à efetividade da intervenção para essa categoria, pode-se destacar que, após a Sessão 18, a porcentagem de ocorrência de CS se tornou ainda menor e se manteve baixa até o final do estudo. Em relação às categorias Correções Parciais (CO) e Correções com Descrições de Consequências (CODC), observou-se que, ao longo do estudo, houve uma redução gradual na porcentagem média de correções (de 8,67\% na linha de base, para 7,67\% na intervenção e para 5,34\% na pós-intervenção). "Para as categorias Elogios Parciais (EP) e Elogios Descritivos (ED), observouse que a maior parte dos elogios feitos pelo treinador, durante a fase de linha de base, era de elogios parciais. Com a intervenção, a porcentagem média de ED aumentou (de 1,64\% na fase de linha de base para 5,50\% na fase de intervenção), principalmente a partir da Sessão 18. Destacam-se as Sessões 18 (13,16\%) e 19 (14,46\%), nas quais se enfatizou a importância do reforço positivo. Após a retirada da intervenção, a porcentagem de ED aumentou (8,43\%).

Em relação aos tipos de exercícios aplicados pelo treinador, observou-se que, durante a linha de base, eles variavam entre exercícios que valiam pontos, exercícios 


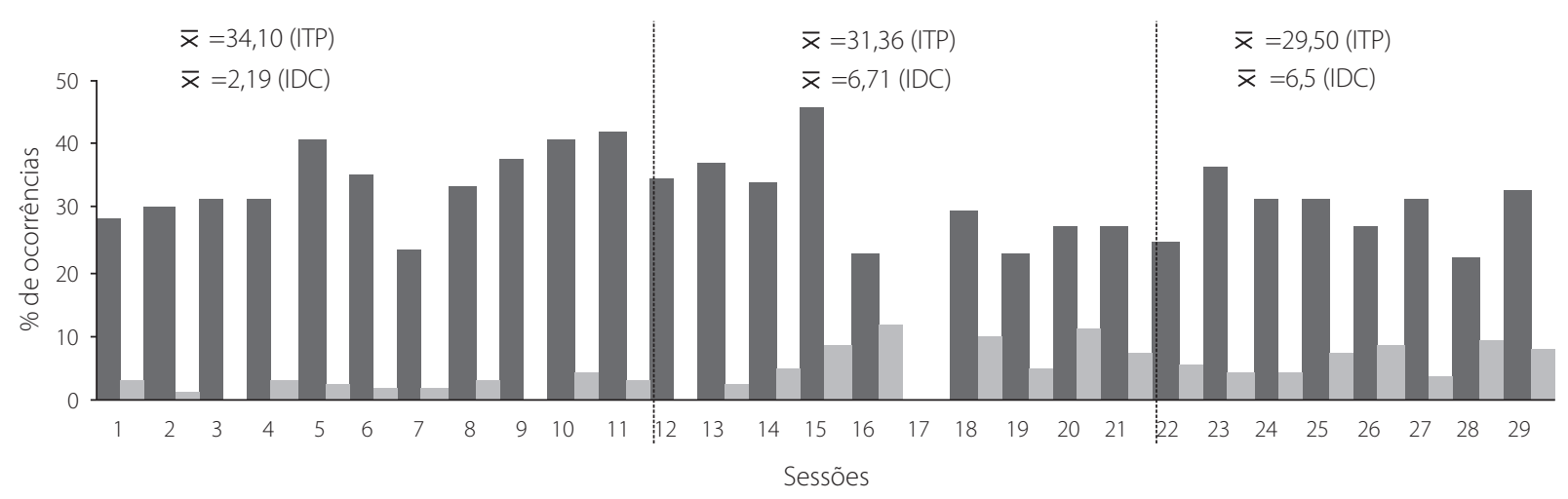

ITP IDC

Encorajamento (ENC)

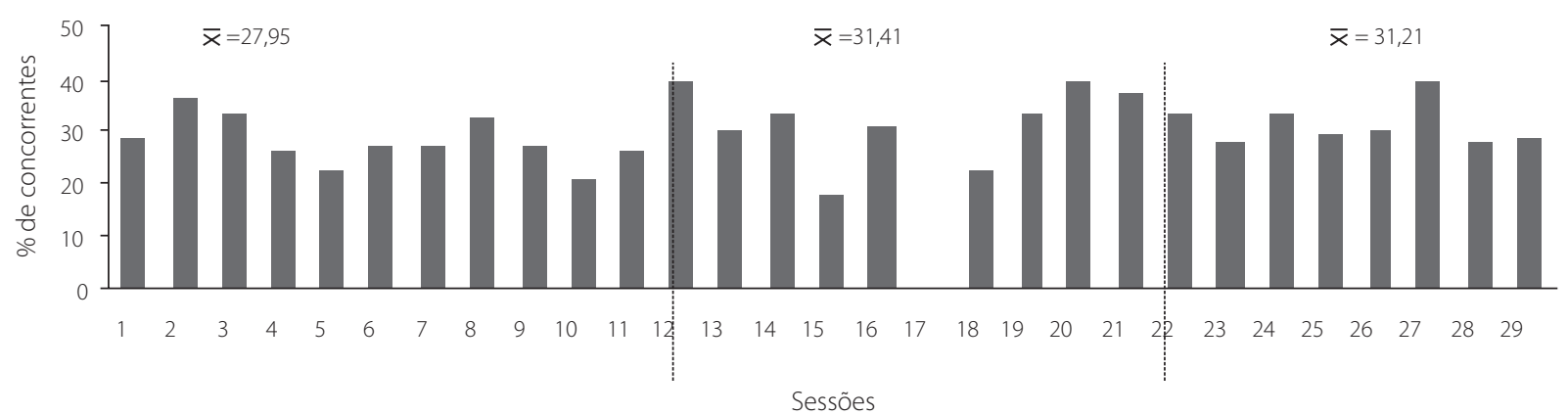

Críticas e Sarcasmo (CS)

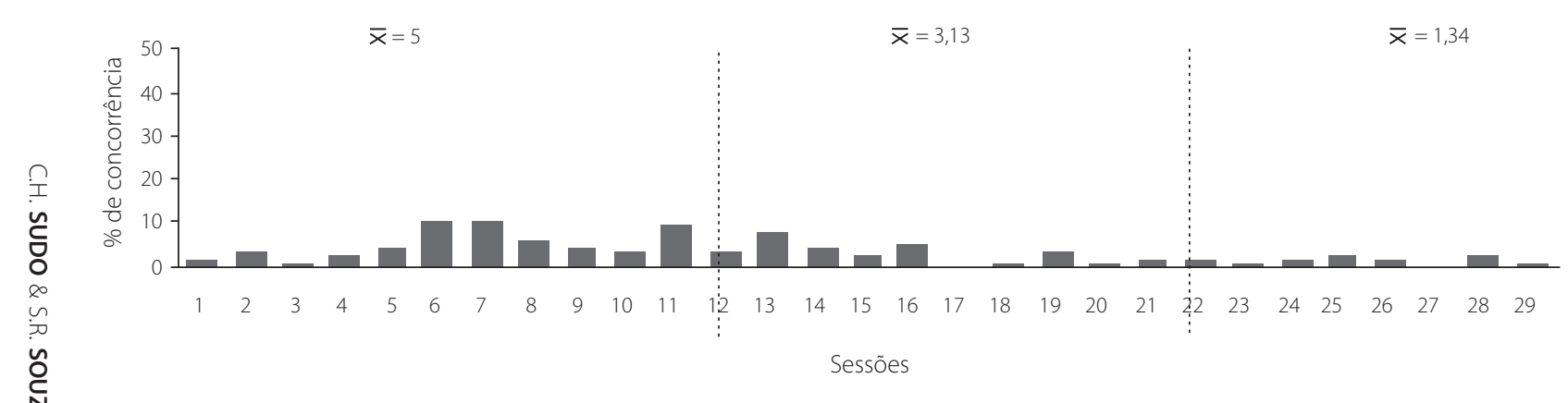

Continuação

Figura 1. Porcentagem de ocorrência das verbalizações do treinador na interação com os atletas, ao longo das três fases do estudo. Londrina 570 (PR), 2006-2007. 


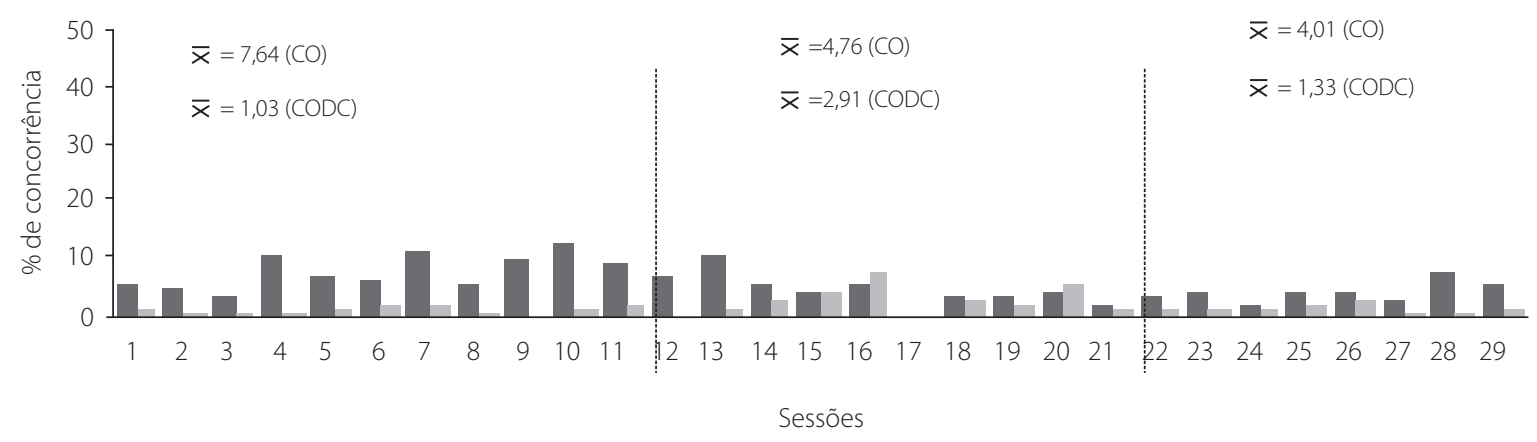

\section{$\square \mathrm{CO} \quad \mathrm{CODC}$}

Elogios Parciais (EP) e Elogios Descritivos (ED)

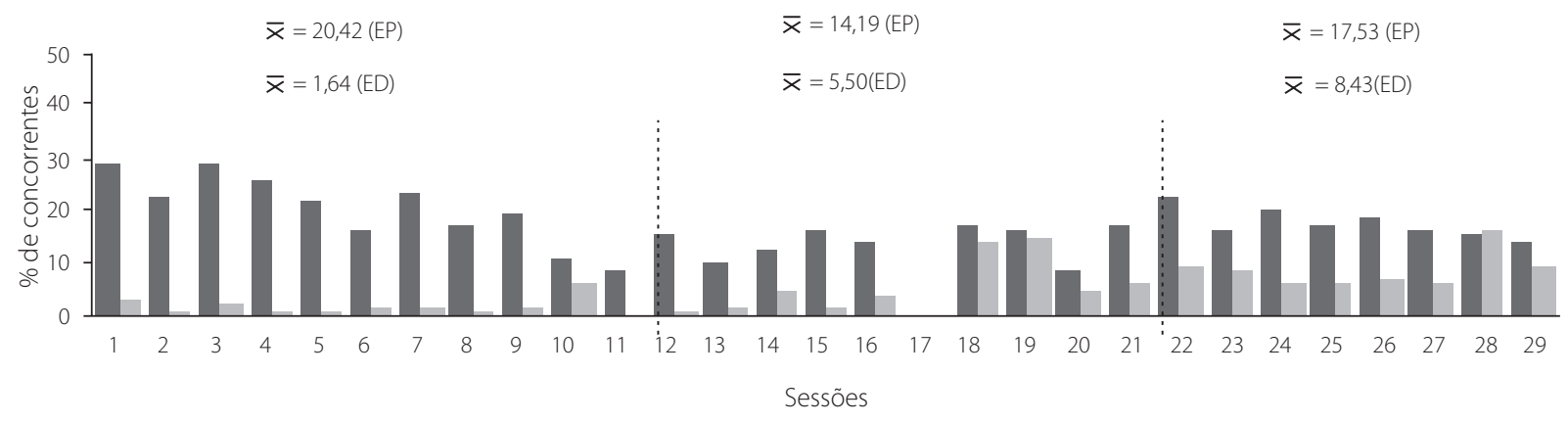

EP $\square \mathrm{ED}$

Figura 1. Porcentagem de ocorrência das verbalizações do treinador na interação com os atletas, ao longo das três fases do estudo. Londrina (PR), 2006-2007.

de Winner', de coordenação motora, de golpes no fundo de quadra, de jogo de rede e de saque. Estes variavam a cada sessão de treino, conforme relato do treinador, na avaliação final:

"Eu ficava muito sob o controle do desempenho deles nosjogos. Se eles fossem jogar e um cara lá ganhasse na regularidade, no fundo de quadra, eu achava que a gente tinha que começar a treinar mais isso" (Sic).

Após a introdução da intervenção, contudo, verificou-se que o treinador incluiu exercícios que se mantiveram regulares por vários treinos, principalmente aqueles que auxiliariam os atletas na execução de seus comportamentos-alvo. A Figura 2 apresenta a porcentagem de exercícios considerados auxiliares para A1 e A2, ao longo do estudo 5 . O comportamento-alvo escolhido para A1 foi: sacar na esquerda e, após a devolução do saque, acelerar um Winner de direita no lado oposto ao do saque. Os critérios de acerto escolhidos foram: bolas dentro do alvo e rápidas, sendo considerados "quase acertos" as bolas perto do alvo e rápidas.

$\boldsymbol{\nabla} \mathbf{\nabla} \boldsymbol{\nabla}$

4 Exercícios de Winner correspondiam a exercícios em que o atleta deveria simular a execução de um golpe que valesse o ponto vencedor, durante a jogada. s A análise dessas categorias durante a Linha de Base foi feita somente após a escolha do comportamento-alvo dos atletas A1 e A2, na fase de intervenção, de modo retroativo. Por isso, não foi possível considerar o critério de estabilidade da linha de base. 
Posteriormente, para A2, o comportamento-alvo escolhido foi: receber um saque, à sua direita, e devolver um Winner cruzado, de direita, no lado do sacador. Os critérios de acerto escolhidos foram: bolas dentro do alvo e rápidas. A escolha do comportamento de $A 2$ foi feita na sexta sessão de intervenção, espontaneamente, sem auxílio da pesquisadora.

Verifica-se que a porcentagem média de exercícios relacionados ao treino do comportamento-alvo dos atletas aumentou de 17,80\% para 78,83\% com a intervenção, e se manteve alta $(63,19 \%)$ mesmo após a retirada dessa. Na décima sessão de intervenção (Sessão 21), o treinador verbalizou:

"Eu vou dar um treino que você vai ver só se eles vão conseguir ou não vai. Eu vou dar bastante dril/6 destes com objetivo de dar winner que eles vão chegar ao final do mês regaçando"(Sic).

Em relação aos comportamentos de registrar desempenho de atletas e de participar dos exercícios junto com eles, a Figura 2 mostra que, com a intervenção, a porcentagem média de registros aumentou de 15,52\% para $64,81 \%$. Ressalta-se que nas Sessões 9 e 10, os atletas tiveram treino de tie-break ${ }^{7}$ e de simulações de jogadas, nos quais a frequência dos registros foi maior devido ao fato de o treinador contar os pontos de cada atleta, durante o tie-break.

Observou-se também, embora de forma não sistematizada, que o treinador começou a usar alvos para os golpes (correntes colocadas no chão, armações de metal em formato de alvo etc.) e recompensas arbitrárias (oferecer refrigerante para quem acertasse o alvo duas vezes), durante os exercícios. Também a partir da quinta sessão de intervenção, o treinador passou a dar feedback do comportamento-alvo dos atletas, ao final de cada treino, o que se repetiu até o final do estudo.

Verificou-se, ainda, que houve mudança no comportamento do treinador em relação a A2, que inicialmente não fora alvo da intervenção. É possível que, devido ao fato de A1 e A2 treinarem juntos, as mudanças de comportamento do treinador ocorreram na interação com ambos. Além disso, os feedbacks que a pesquisadora dava sobre o comportamento do treinador, na intervenção, não discriminavam apenas a interação do treinador com A1. O mesmo ocorreu com relação aos registros de desempenho de $\mathrm{A} 2$.

Comportamentos do Treinador Durante os Treinos

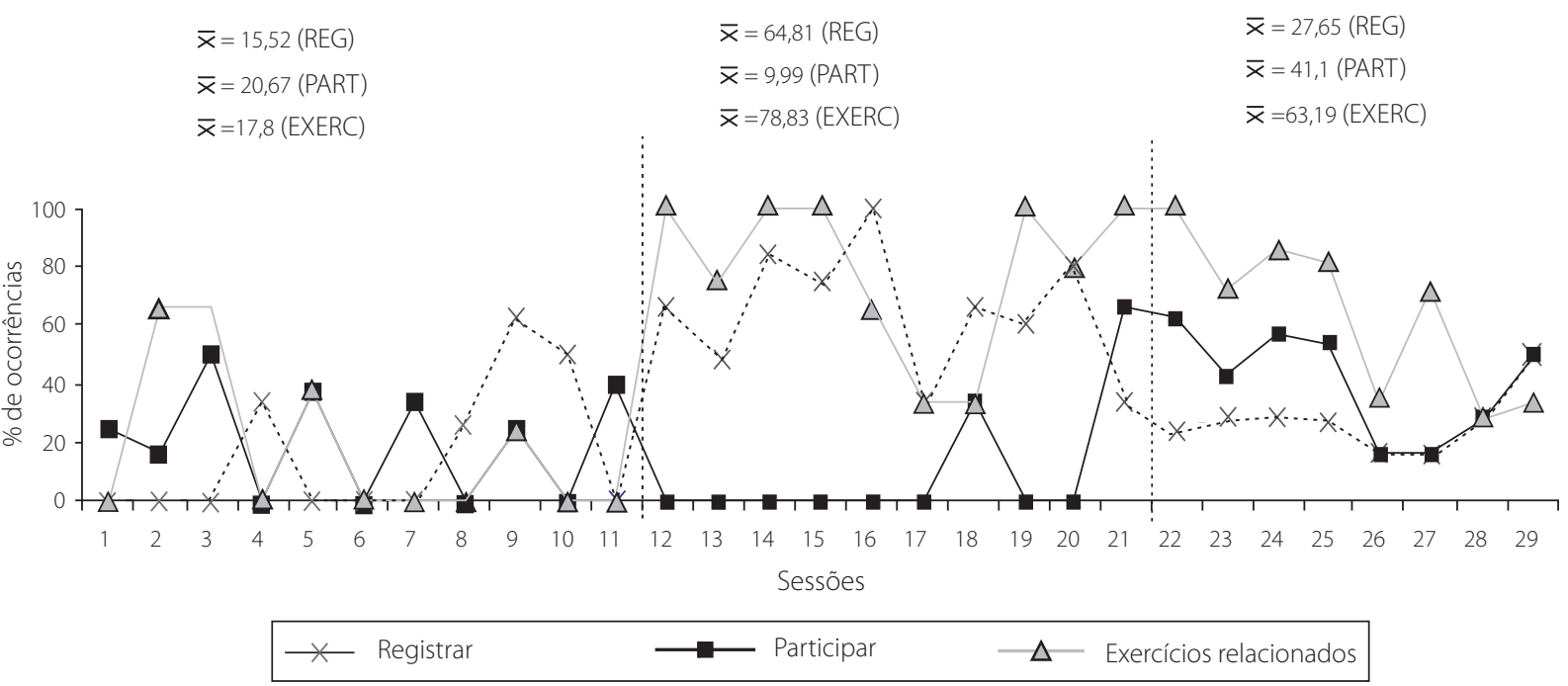

Figura 2. Porcentagem de ocorrência de exercícios relacionados ao treino do comportamento-alvo, e porcentagem de compor-tamentos de registrar desempenho dos atletas e de participar diretamente dos exercícios, fazendo-os com os atletas, ao longo do estudo. Londrina (PR), 2006-2007.

- Drill é uma expressão utilizada pelo treinador para referir-se ao uma série sequencial de exercícios.

5727 Tie-break é a expressão que representa a simulação de jogo em que são contados os pontos corridos, sem se considerarem as vantagens. 


\section{Discussão}

Os resultados sugerem que, após a intervenção, o treinador foi capaz de: registrar e montar a linha de base do desempenho inicial do atleta, sistematizar os exercícios do treino e elaborar etapas de evolução do desempenho dos comportamentos-alvo. Ele começou a fornecer instruções mais completas e a descrever os erros e acertos dos atletas, além do que reduziu as correções parciais e as críticas e sarcasmos dirigidos a eles. Além disso, o treinador forneceu feedback dos comportamentos-alvo dos atletas e registrou outros comportamentos que não aqueles selecionados para este trabalho. Por fim, o treinador passou a utilizar recompensas arbitrárias em várias situações do treino.

Em relação às verbalizações do treinador, foram mais frequentes as instruções técnicas parciais, encorajamentos e elogios parciais. Pesquisas (Lima \& Souza, 2009; Macedo \& Souza, 2009) mostram resultados similares. No entanto, conforme análise do comportamento, a instrução que descreve os antecedentes e consequentes de uma resposta, de forma clara, é mais efetiva para a mudança de comportamento do que as instruções que descrevem apenas a resposta, ou seja, as parciais (Martin, 2001). No presente estudo, o aumento de instrução com a descrição de consequências, principalmente a partir da Sessão 15, pareceu relevante.

Sugere-se que as intervenções dos passos 1 e 2 , principalmente, possam ter auxiliado o treinador no fornecimento de instruções mais completas, visto que possibilitaram uma melhor observação do comportamento do atleta, bem como dos seus antecedentes e consequentes. Isso pode estar relacionado, também, ao aumento dos elogios descritivos e à redução das correções e das criticas ou sarcasmo, por serem respostas concorrentes.

A partir da sessão 16 (passo 2), o treinador passou a dar feedback do comportamento-alvo, ao final dos treinos. Não obstante algumas formas de feedback poderem reforçar comportamentos - embora seja impossível defini-los antecipadamente - seria interessante considerar que o feedback pode estar relacionado à efetividade das metas porque auxilia na identificação de variáveis relacionadas aos acertos e erros, favorecendo orientações mais efetivas (Austin, Weatherly \& Gravina, 2005; Mouratidis, Lens \& Vansteenkiste, 2010). De fato, isso foi observado no presente estudo.
Também Gamba (2007); Humee Crossman (1992); Sudo e Souza (2009) mostram a importância de identificar diferentes fontes de reforçadores para comportamentos dos atletas, como música (Hume \& Crossman, 1992), entrega de prêmios escolhidos por eles (Gamba, 2007) e participação em uma atividade desejada, ao final do treino (Martin, 2001). Neste estudo, o treinador foi orientado a fazer o levantamento de recompensas de interesse de seus atletas e, também, a observar os efeitos de diferentes tipos de consequências sobre seus comportamentos, durante os treinos. A contagem de pontos trocáveis por refrigerantes ou por permissão para descanso parece ter influenciado positivamente o comportamento dos atletas nos treinos. Observações não sistemáticas mostraram que, nessas ocasiões, os atletas reclamavam menos e acertavam mais.

Verificou-se que os passos da intervenção tinham como função capacitar o treinador a, mais do que estabelecer uma meta, propiciar o rearranjo das contingências de treino com vistas a aumentar a probabilidade de que o comportamento-alvo fosse bem sucedido. Apesar dos registros dos comportamentos dos atletas terem sido feitos pelo treinador e sem nenhum controle experimental - o que inviabiliza afirmações quanto aos efeitos da intervenção sobre esses comportamentos - verificou-se que as mudanças observadas tanto nas verbalizações do treinador quanto no rearranjo do ambiente de treino parecem ter auxiliado na melhora do comportamento-alvo dos atletas. Tais mudanças refletiram, também, na percepção que os atletas tinham de seu comprometimento nos treinos. A2, na avaliação final, verbalizou: "Isso nos dá mais concentração efaz a gente ter mais vontade de acertar. Me deu mais coragem nos jogos, me senti mais confiante" (sic).

Muitos trabalhos se propuseram a ensinar treinadores a serem mais efetivos no uso de instruções, correções, elogios etc. (Cruz et al., 2011; Martens, 1990; Smith, Smoll \& Curtes, 1983), ou ainda, no gerenciamento de seus treinos (Gilbert \&Trudel, 1999). Mas, destaca-se neste trabalho o fato de os comportamentos do treinador terem sido categorizados e observados sistematicamente e avaliados antes, durante e após a intervenção. Para o ensino das habilidades envolvidas nos passos do estabelecimento de metas, utilizaram-se, neste estudo, instruções, modelos, video feedback e modelagem 
direta de comportamentos nas sessões de treino, com feedback dos comportamentos do treinador em ambiente natural de treino. Como resultado, verificou-se que:

1) As mudanças obtidas durante a intervenção, principalmente as relacionadas à categoria das verbalizações, mantiveram-se após a retirada da intervenção.

2) Conquanto a intervenção tenha sido conduzida com o treinador na interação com apenas um de seus atletas, as mudanças ocorridas em sua interação com A1 ocorreram também em sua interação com A2, mesmo sem o auxílio da pesquisadora. Apesar da possibilidade de a intervenção ter reforçado comportamentos do treinador na interação com A2, uma vez que o treinador interagia com ambos os atletas ao mesmo tempo, ao final do estudo, ele apresentou à pesquisadora um programa de metas que havia estabelecido para outro grupo de atletas, o que mostra a generalização desse comportamento para diferentes contextos.

\section{Considerações Finais}

Os dados obtidos sugerem que o estabelecimento de metas funciona como uma nova contingência de ensino não apenas para os atletas, mas também para o treinador, que passa a ser mais criterioso em suas instruções, correções, críticas, organização do treino etc. Isso significa que uma meta como "faça 10 saltos corretamente" só é efetiva se ela puder, de fato, alterar a probabilidade de o salto ocorrer e ser reforçado. Essa análise se mostra importante, pois como aponta Cruz et al. (2011), estudos sobre estabelecimento de metas tendem a se prender a análises topográficas dos comportamentos e a desconsiderar a análise de sua função.

Em vista dessa conclusão, surgem algumas considerações: compreensão acerca dos aspectos que, de fato, tornam o estabelecimento de metas efetivo, pode gerar conclusões precipitadas na avaliação dessa estratégia.

574 Assim, futuros estudos poderiam avaliar os efeitos do estabelecimento de metas e/ou de outras estratégias, a partir de análises funcionais dos comportamentos-alvo e não, apenas, a partir de análises topográficas (Corrêa \& Souza Júnior, 2009; Marinho, 2010; Neiva, 2010). Isso requer ampliar a análise da influência de comportamentos de treinadores sobre o desempenho atlético, como já tem sido sugerido em outros estudos (Brandão \& Carchan, 2010).

2) Futuras pesquisas poderiam utilizar delineamentos de caso único (Martin, Thompson \& Regher, 2004), avaliar os efeitos de procedimentos específicos, separadamente, e não em pacotes de intervenção como feito neste estudo, e investigar os efeitos das intervenções com treinadores sobre os comportamentos dos atletas - a partir de observações diretas de seu comportamento, e não somente a partir de medidas indiretas.

3) Futuros estudos poderiam investigar os efeitos do ensino de uma habilidade específica sobre o aprendizado das outras habilidades. Por exemplo, sugere-se que o ensino de observação e registro sistemáticos aos treinadores possibilite que eles forneçam orientações mais completas, mesmo que não tenham sido diretamente orientados quanto a estes últimos.

4) Por fim, futuros estudos poderiam envolver um maior número de treinadores e de atletas de outras modalidades como, por exemplo, os de esportes coletivos.

\section{Referências}

Austin, J., Weatherly, N., \& Gravina, N. E. (2005). Using task clarification, graphic feedback e verbal feedback to increase closing-task completion in a privately owned restaurant. Journal of Applied Behavior Analysis, 38 (1), 117-120.

Brandão, M. R. F., \& Carchan, D. (2010). Comportamento preferido de liderança e sua influência no desempenho dos atletas. Motricidade, 6 (1), 53-69.

Brobst, B., \& Ward, P. (2002). Effects of public posting, goal setting and oral feedback on the skill of female soccer players. Journal of Applied Behavior Analysis, 35 (3), 247-257.

Burton, D., Weinberg, R., Yukelson, D., \& Weingand, D. (1998). The goal effectiveness paradox in sport: examining the goal practices of collegiate athletes. The Sport Psychologist, 12 (4), 404-418.

Cedra, C., \& Sério, T. M. A. P. (2008). O treinamento do lance livre no basquetebol. Revista Brasileira de Psicologia do Esporte, 2 (1), 1-28. 
Coatsworth, J. D., \& Conroy, D. E. (2006). Enhancing the self-esteem of youth swimmers through coach training: gender and age effects. Psychology of Sport and Exercise, 7, 173-192.

Conde, C., Almagro, B. J., Sáenz-López, E., \& Castillo, E. (2009). Intervention and evaluation of the motivational climate transmitted by a basketball coach. Revista de Psicología del Deporte, 18 (Suppl.), 357-361.

Corrêa, U. C. \& Souza Jr., O. P. (2009). Effects of goal difficulty and temporality in motor skill acquisition using the Bachman ladder. Perceptual and Motor Skill, 109, 817-823.

Cruz, J. F., Dias, C., Gomes, R., Alves, A., Sá, S., Viveiros, I., et al. (2001). Um programa de formação para a eficácia dos treinadores da iniciação e formação desportiva. Análise Psicológica, 1 (19), 171-182.

Cruz, J., Torregrosa, M., Sousa, C., Mora, A., \& Viladrich, C. (2011). Efectos conductuales de programas personalizados de asesoramiento a entrenadores en estilo de comunicación y clima motivacional. Revista de Psicología del Deporte, 20 (1),179-195.

Cunha, G. B., Mesquita, I. M. R., Rosado, A. F. B., Sousa, T., \& Pereira, P. (2010). Necessidades de formação para o exercício profissional na perspectiva do treinador de Futebol em função da sua experiência e nível de formação. Motriz, 16 (4), 31-41.

Demmers, G., Woodburn, A. J., \& Savard, C. (2006). The development of an undergraduate competency-based coach education program. The Sport Psychologist, 20 (2), 162-173.

Gamba, J.F. (2007). Efeitos de um programa deestabelecimento de metas efeedbackna execução de fundamentos esportivos realizados por crianças praticantes de ginástica artística. Dissertação de mestrado não-publicada. Programa de Mestrado em Análise do Comportamento, Universidade Estadual de Londrina.

Getz, G. E., \& Rainey, D. W. (2001). Flexible short-term goal and basketball shooting performance. Journal of Sport Behavior, 24 (1), 31-41.

Gilbert, W., \& Trudel, P. (1999). An evaluation strategy for coach education programs. Journal of Sport Behavior, 22 (2), 234-250

Gonzáles, J. L. (2001). El estabelecimento de objetivos como técnica motivacional. In J. L. González; C. G. Rodriguez \& G. M. García (Orgs.), Manual de prácticas de psicología deportiva: las ciencias del comportamiento deportivo, prácticas de entrenamiento mental (pp.97-109). Madri: Biblioteca Nuevo.

Hume, K. M., \& Crossman, J. (1992). Musical reinforcement of practice behaviors among competitive swimmers. Journal of Applied Behavior Analysis, 25, 665-670.

Kazdin, A. E. (1982). Single case research designs: methods for clinical and applied settings. New York: Oxford University Press.

Lima, P. V., \& Souza, S. R. (2009). O treinador esportivo e as categorias de base: os efeitos de uma intervenção sobre os comentários feitos por treinadores durante eventos competitivos. In S. R. Souza \& V. B. Haydu (Orgs.), Psicologia comportamental aplicada: avaliação e intervenção na área do esporte, clínica, saúdeeeducação (pp.213-229). Londrina: EDUEL.

Macedo, R. H., \& Souza, S. R. (2009). Análise dos comentários dos treinadores durante Competição Infantil. In S. R. Souza \& V. B. Haydu (Orgs.), Psicologia comportamental aplicada: avaliação e intervenções na área do esporte clinica, saúde e educação (197-210). Londrina: EDUEL.

Mallett, C., \& Côté, J. (2006). Beyond winning and losing: guidelines for evaluating high performance coaches. The Sport Psychologist, 20, 213-221.

Marinho, N. F. S. (2009). Efeito da dificuldade da meta na aprendizagem motora em sujeitos orientados à tarefa. Dissertação de mestrado não-publicada, Escola de Educação Física, Fisioterapia e Terapia Ocupacional, Universidade Federal de Minas Gerais, Belo Horizonte.

Martens, R. (1990). Successful coaching ( $2^{\text {nd }}$ ed.). American coaching effectiveness program leader level course. Illinois: Human Kinectics.

Martin, G. L. (2001). Consultoria em psicologia do esporte: orientações práticas em análise do comportamento. Campinas: Instituto de Análise do Comportamento.

Martin, G. L., Thompson, K., \& Regher K. (2004). Studies using single-subject designs in sport psychology: 30 years of research. The Behavior Analyst, 27 (2), 263-280.

Mellalieu, S. D., Hanton, S., \& O'Brien, M. (2006). The effects of goal setting on rugby performance. Journal of Applied Behavior Analysis, 39, 257-261.

Mouratidis, A., Lens, W., \& Vansteenkiste, M. (2010). How you provide corrective feedback makes a difference: the motivating role of communicating in an autonomysupporting way. Journal of Sport and Exercise Psychology, 32 (5), 619-637.

Mora, A., Cruz, J., \& Torregrosa, M. (2009). Effects of a training program in communication styles of basketball coaches. Revista de Psicología del Deporte, 18 (Suppl.), 299-302.

Neiva, J. F. O. (2010). Estabelecimento de metas e ansiedade traço na aquisição de habilidades motoras. Dissertação de mestrado não-publicada, Escola de Educação Física e Esporte, Universidade de São Paulo.

Ramos, V., Graça, A. B. S., Nascimento, J. V., \& Silva, R. (2011). A aprendizagem profissional: as representações de treinadores desportivos de jovens: quatro estudos de caso. Motriz, 17 (2), 280-291.

Rushall, B. S. \& Smith, K. C. (1983). The modification of the quality and quantity of behavior categories in a swimming coach. In G. L. Martin \& D. Hrycaiko (Orgs.), Behavioral modification and coaching:principles, procedures and research (pp.321-336). Illions: Charles C. Thomas Publishers.

Skinner, B. F. (1953). Ciência e comportamento humano. São Paulo: Martins Fontes.

Smith, R. E., \& Smoll, F. L. (1997). Coaching the coaches: youth sports as a scientific an applied behavioral setting. Current Directions in Psychological Science, 6 (1), 16-21.

Smith, R. E., \& Smoll, F. L. (2011). Cognitive-behavioral coach training: a translational approach to theory, research and intervention. In J. K. Luiselli \& D. D. Reed (Orgs.), Behavioral 
sport psychology: evidence-based approaches to performance enhancement (pp.227-248). New York: Springer Science Business Media.

Smith, R. E., Smoll, F. L., \& Cumming, S. P. (2007). Effects of a motivational climate intervention for coaches on young athletes' sport performance anxiety. Journal of Sport \& Exercise Psychology, 29 (1), 39-59.

Smith, R. E., Smoll, F. L., \& Curtis, B. (1983). Coach effectiveness training: a cognitive-behavioral approach to enhancing relationship skills in youth sport coaches. In G. L. Martin \& D. Hrycaiko (Orgs.), Behavioral modification and coaching. principles, procedures and research (pp.337-360). Illions: Charles C. Thomas Publishers.

Smith, R. E., Smoll, F. L., \& Hunt, B. (1977). A system for the behavioral assessment of athletic coaches. Research Quaterly, 48 (2), 401-407.

Smoll, F. L., Smith, R. E., \& Curtis, B. (1983). Behavioral guidelines for youth sport coaches. In G. L. Martin \& D. Hrycaiko (Org.), Behavioral modification and coaching. principles, procedures and research (pp.61-66). Illions: Charles C. Thomas Publishers.

Souza, S. R., Sudo, C. H., Gamba, J. F., Oliveira, S. D., \& Teixeira, B. F. (2009). Estabelecimento de metas e feedback: uma intervenção com atletas de uma equipe feminina de futsal da categoria adulto. In S. R. Souza \& V. B. Haydu (Orgs.), Psicologia comportamental aplicada: avaliação e intervenção na área do esporte, clínica, saúde e educação (pp.179-197). Londrina: EDUEL.

Sousa, C., Cruz, J., Torregrosa, M., Vilches, D., \& Viladrich, C. (2006). Evaluación conductual y Programa de
Asesoramiento Personalizado a Entrenadores (PAPE) de desportistas. Revista de Psicología del Deporte, 15 (2), 263-278.

Sudo, C. H., \& Souza, S. R. (2009). Efeitos do uso do estabelecimento de metas sobre o desempenho de atletas de tênis. In R. C. Wielenska (Org.), Sobre comportamento e cognição: desafios, soluções e questionamentos (pp.219-229). Santo André: ESETec.

Swain, A., \& Jones, G. (1995). Effects of goal-setting interventions on selected basketball skills: a singlesubject design. Research Quarterly Exercise and Sport, 66 (1), 51-63.

Ugrinowitsh, H., \& Dantas, L. E. P. B. T. (2002). Efeito do estabelecimento de metas na aprendizagem do arremesso do basquetebol. Revista Brasileira de Ciências do Desporto, 2 (5), 58-63.

Ward, P. (2011). Goal setting and performance feedback. In J. K. Luiselli \& D. D. Reed (Org.), Behavioral sport psychology: evidence-based approaches to performance enhancement. (pp.99-112). New York, US: Springer Science Business Media.

Ward, P., \& Carnes, M. (2002). Effects of posting self-set goals on collegiate football players' skill execution during practice and games. Journal of Applied Behavior Analysis, $35,1-12$.

Weinberg, R. S., \& Gould, D. (2001). Fundamentos da psicologia do esporte e do exercício ( $2^{\mathrm{a}}$ ed.). Porto Alegre: Artmed.

Recebido em: 11/11/2010

Versão final em: 22/12/2011

Aprovado em: 16/3/2012 